\title{
THE INTEGRAL GEOMETRY DEFINITION OF ARC LENGTH FOR TWO-DIMENSIONAL FINSLER SPACES
}

\author{
BY \\ O. G. OWENS
}

Introduction. A curve $C^{*}$ in a 2-dimensional Finsler space, metrized by $d s=F(x, y, d x, d y)$, is admissible if it is a simple, closed, regular, extremal convex curve, where extremal convexity means locally $C^{*}$ lies in the closure of a component (side) of any tangent geodesic.

A conjecture of Hans Lewy is that an "integral geometry" relation, proved by Legendre for euclidean space, can be generalized to the following:

Theorem. Specify the geodesics by suitable parameters $\theta, p$ and let $D$ denote the cartesian region consisting of all points $(\theta, p)$ for which the corresponding geodesic intersects $C^{*}$. Then there exists a contact transformation $(x, y, d x, d y)$ $\rightarrow(\theta, p, d \theta, d p)$ and a density function $\sigma(\theta, p)$ such that the Finsler length of any admissible $C^{*}$, extremal convex in the large, is

$$
\oint_{C^{*}} F(x, y, d x, d y)=\iint_{D} \sigma(\theta, p) d p d \theta .
$$

The integral equality (1) will be established for admissible $C^{*}$ contained in a region $U$, where: (a) Certain regularity and boundedness conditions hold for $F$ and specified partial derivatives of $F$ on $U$; for example, $F$ is to define a regular variational problem. (b) The geodesics of $F$ satisfy a "field hypothesis," see Part II, (3.4). (c) An "embedment hypothesis" holds for $C^{*}$, see Part II, (9.1).

Assuming the geodesics are straight lines, Eberhard Hopf devised an elegant proof of (1) and with no restriction as to the sign of $F$. I thank Professor Hopf for allowing me to present his proof as Part I of this paper. In Part II the author resolves the technical difficulties met in generalizing the Hopf procedure and establishes the theorem and a generalization applicable to $C^{*}$ not necessarily extremal convex in the large.

\section{PART I}

We shall assume that the Finsler metric, $d s=F(x, y, d x, d y)$, has straight linès for geodesics, is of class $C^{3}$ in its arguments, and that $U$ is any convex region on which $F$ is defined.

1. Variation of Finsler length. Impose the restriction, later to be removed, that $C^{*}$ encloses the origin of coordinates, and let $\phi$ denote the positive angle formed by the directed tangent with the polar axis. Then,

Received by the editors October 28, 1951 and, in revised form, January 17, 1952. 


$$
\frac{d x}{d t}=\cos \phi, \quad \frac{d y}{d t}=\sin \phi, \quad \frac{d y}{d x}=\tan \phi,
$$

where $d t=\left((d x)^{2}+(d y)^{2}\right)^{1 / 2}$. Hence, as $F(x, y, d x, d y)$ is homogeneous of degree one in its differentials,

$$
d s=F\left(x, y, \frac{d x}{d t}, \frac{d y}{d t}\right) d t \equiv L(x, y, \phi) d t,
$$

defining $L(x, y, \phi)$.

If $\delta$ denotes the first väriation, then

$$
\delta d t=(\delta d x) \cos \phi+(\delta d y) \sin \phi .
$$

Furthermore, since

$$
\sec ^{2} \phi \delta \phi=\frac{d x(\delta d y)-d y(\delta d x)}{(d x)^{2}}
$$

it follows that

$$
d t \delta \phi=(\delta d y) \cos \phi-(\delta d x) \sin \phi .
$$

Assuming fixed end points $P_{0}$ and $P_{1}$, we now compute the variation of

$$
I=\int_{P_{0}}^{P_{1}} L(x, y, \phi) d t .
$$

By means of (1.3), (1.4), and the vanishing of the variations at $P_{0}$ and $P_{1}$, we find

$$
\begin{aligned}
\delta I= & \int_{P_{0}}^{P_{1}}\left[L_{x} d t-d(L \cos \phi)+d\left(L_{\phi} \sin \phi\right)\right] \delta x \\
& +\int_{P_{0}}^{P_{1}}\left[L_{y} d t-d(L \sin \phi)-d\left(L_{\phi} \cos \phi\right)\right] \delta y .
\end{aligned}
$$

Therefore, if

$$
X \equiv L_{x} d t-d\left[-L_{\phi} \sin \phi+L \cos \phi\right]
$$

and

$$
Y \equiv L_{y} d t-d\left[L_{\phi} \cos \phi+L \sin \phi\right]
$$

then

$$
\delta I=\int_{P_{0}}^{P_{1}}[X \delta x+Y \delta y] .
$$

This result also holds for closed curves, $P_{0} \equiv P_{1}$, as well as curvilinear seg- 
ments bounded by distinct end points.

2. Simplification of $\delta I$. The differential forms $X$ and $Y$ satisfy the following relations:

$$
\begin{aligned}
X \cos \phi+Y \sin \phi & =0, \\
X \sin \phi-Y \cos \phi & =S(x, y, \phi) d t+T(x, y, \phi) d \phi,
\end{aligned}
$$

where

$$
T(x, y, \phi) \equiv L+L_{\phi \phi}
$$

and

$$
S(x, y, \phi) \equiv L_{x \phi} \cos \phi+L_{y \phi} \sin \phi+L_{x} \sin \phi-L_{y} \cos \phi .
$$

A consequence of (2.3) and (2.4) is

$$
\frac{\partial S}{\partial \phi}=\frac{\partial T}{\partial x} \cos \phi+\frac{\partial T}{\partial y} \sin \phi .
$$

Furthermore, as the extremals of the variational problem, $I=$ minimum, are straight lines, $X=Y=0$, whenever $d \phi=0$, which implies

$$
S(x, y, \phi) \equiv 0 .
$$

Thus, because of (2.1), (2.2), and (2.6),

$$
X=T \sin \phi d \phi, \quad Y=-T \cos \phi d \phi .
$$

Substituting (2.7) into (1.9) gives

$$
\delta I=\oint_{c^{*}} T[\delta x \sin \phi-\delta y \cos \phi] d \phi .
$$

3. Identity of $I$ with double integral. Since the closed convex curve $C^{*}$ encircles the origin, there is a unique $p=p(\phi)$ such that $C^{*}$ is the envelope of its tangent lines:

$$
x \sin \phi-y \cos \phi=p(\phi) .
$$

The parametric equations of $C^{*}$ are

$$
\begin{aligned}
& x=p \sin \phi+p^{\prime} \cos \phi, \\
& y=-p \cos \phi+p^{\prime} \sin \phi,
\end{aligned}
$$

$$
\left(p^{\prime} \equiv \frac{d p}{d \phi}\right)
$$

Thus, because of (2.5), (2.6), and (3.2),

$$
\frac{\partial T}{\partial p^{\prime}}=\frac{\partial T}{\partial x} \cos \phi+\frac{\partial T}{\partial y} \sin \phi=\frac{\partial S}{\partial \phi} \equiv 0,
$$

so $T=T(\phi, p)$ is not a function of $p^{\prime}$. Now, let the variation of $C^{*}$ be that obtained by varying the support function $p$. Then, 


$$
\delta p=\delta x \sin \phi-\delta y \cos \phi,
$$

and (2.8) becomes

$$
\delta I=\int_{0}^{2 \pi} T \delta p d \phi=\delta \iint_{D} T(\phi, p) d p d \phi,
$$

where $D$ consists of all points $(\phi, p)$ for which $[0 \leqq \phi \leqq 2 \pi ; 0 \leqq p \leqq p(\phi)]$, or all points $(\phi, p)$ such that the corresponding line (3.1) intersects $C^{*}$. Consequently, as the two integrals in (3.5) have equal variations and as both vanish when $C^{*}$ is contracted to the origin, they are equal. That is,

$$
I \equiv \oint_{c^{*}} L(x, y, \phi) d t=\iint_{D}\left[L+L_{\phi \phi}\right] d p d \phi,
$$

where the integrand of the double integral depends only on the variables $(\phi, p)$.

Consider now the case that $C^{*}$ does not enclose the origin. Let $\alpha$ and $\beta$ be the smaller and larger, respectively, of the positive angles formed by the polar axis and the two directed tangents to $C^{*}$ from the origin. The map of $C^{*}$ under (3.1) will consist of two separate curvilinear segments:

$$
p=p_{1}(\phi), \quad p=p_{2}(\phi),
$$

where $p_{1}(\phi) \leqq p_{2}(\phi)$ and $\alpha \leqq \phi \leqq \pi+\beta$. If $D$ is taken as the set of points $(\phi, p)$ satisfying the inequalities

$$
\alpha \leqq \phi \leqq \pi+\beta, \quad p_{1}(\phi) \leqq p \leqq p_{2}(\phi),
$$

then (3.6) still holds and has the same "integral geometry" interpretation.

\section{PART II}

We shall assume that the Finsler metric, $d s=F(x, y, d x, d y)$, is of class $C^{3}$ in its arguments and defines a regular variational problem on a region $U$ which is extremal convex in the large. Furthermore, on $U$, a "field hypothesis," (3.4), is satisfied by the geodesics and an "embedment hypothesis," (9.1), by the admissible curves $C^{*}$.

1. The Euler equations. Any geodesic $C:[x(t), y(t)]$ of the space is a solution of the Euler equations:

$$
F_{x}-\frac{d F_{\dot{x}}}{d t}=0, \quad F_{y}-\frac{d F_{\dot{y}}}{d t}=0,
$$

where $(x, y, \dot{x}, \dot{y}) \equiv(x(t), y(t), d x / d t, d y / d t)$. Furthermore, the homogeneity condition

$$
F(x, y, k \dot{x}, k \dot{y})=k F(x, y, \dot{x}, \dot{y})
$$

implies the following: 


$$
\begin{aligned}
F_{1}(x, y, \dot{x}, \dot{y}) & \equiv \frac{F_{\dot{x} \dot{x}}}{\dot{y}^{2}}=-\frac{F_{\dot{x} \dot{y}}}{\dot{x} \dot{y}}=\frac{F_{\dot{y} \dot{y}}}{\dot{x}^{2}}, \\
F_{1}(x, y, k \dot{x}, k \dot{y}) & =k^{-3} F_{1}(x, y, \dot{x}, \dot{y}) .
\end{aligned}
$$

Hence, for regular curves $C$, that is,

$$
\rho \equiv\left(\dot{x}^{2}+\dot{y}^{2}\right)^{1 / 2} \neq 0,
$$

the Euler equations are equivalent to the single equation:

$$
(\dot{x} \ddot{y}-\dot{y} \ddot{x}) F_{1}+F_{x \dot{y}}-F_{\dot{x} y}=0
$$

Thus, if

$$
G(x, y, \dot{x}, \dot{y}) \equiv\left(F_{y \dot{x}}-F_{x \dot{y}}\right) / F_{1},
$$

which implies the homogeneity relation

$$
G(x, y, k \dot{x}, k \dot{y})=k^{3} G(x, y, \dot{x}, \dot{y}),
$$

the differential equation (1.6) is equivalent to

$$
\ddot{x}=-\dot{y} G(x, y, \dot{x}, \dot{y}), \quad \ddot{y}=+\dot{x} G(x, y, \dot{x}, \dot{y}),
$$

the parametrization $\rho=1$ being assumed.

From(1.8) follows

$$
G F_{1 \dot{y}}+F_{x \dot{y} \dot{y}}-F_{y \dot{x} \dot{y}}+G_{\dot{y}} F_{1}=0, \quad G F_{1 \dot{x}}+F_{x \dot{x} \dot{y}}-F_{\dot{x} \dot{x} y}+G_{\dot{x}} F_{1}=0,
$$

which, because of (1.4), can be written as

(1.11) $G F_{1 \dot{y}}+\dot{x}^{2} F_{1 x}+\dot{x}_{\dot{y}} F_{1 y}+G \dot{y} F_{1}=0, G F_{1 \dot{x}}-\dot{x} \dot{y} F_{1 x}-\dot{y}^{2} F_{1 y}+G_{\dot{x}} F_{1}=0$.

Thus, since $\rho=1$ by hypothesis,

$$
G\left(\dot{x} F_{1 \dot{y}}-\dot{y} F_{1 x}\right)+\dot{x} F_{1 x}+\dot{y} F_{1 y}+\left(\dot{x} G_{\dot{y}}-\dot{y} G_{\dot{x}}\right) F_{1}=0 .
$$

2. Variation of Finsler length. For a simple, regular arc $C:[(x(t), y(t))$, $\left.t_{0} \leqq t \leqq t_{1}\right]$, bounded by prescribed end points $P_{0} \equiv\left(x\left(t_{0}\right), y\left(t_{0}\right)\right)$ and $P_{1}$ $\equiv\left(x\left(t_{1}\right), y\left(t_{1}\right)\right)$, the variation of the Finsler length

$$
I=\int_{t_{0}}^{t_{1}} F(x, y, \dot{x}, \dot{y}) d t
$$

is

$$
\delta I=\int_{t_{0}}^{t_{1}}\left\{\left[F_{x}-\frac{d F_{\dot{x}}}{d t}\right] \delta x+\left[F_{y}-\frac{d F_{\dot{y}}}{d t}\right] \delta y\right\} d t,
$$

where we assume that

$$
F_{\dot{x}}^{2}+F_{\dot{y}}^{2}<A,
$$


a uniform bound for all $\rho \neq 0$. Moreover, as (2.2) also holds for closed arcs, that is, $P_{0}=P_{1}$,

$$
\delta I=\oint_{C}\left[(\dot{x} \ddot{y}-\dot{y} \ddot{x}) F_{1}+F_{x \dot{y}}-F_{\dot{x} v}\right][d y \delta x-d x \delta y]
$$

for closed curves $C$. Now, because of (1.9), (2.4) becomes

$$
\delta I=\oint_{C}[(\dot{x} \ddot{y}-\dot{y} \ddot{x})-G(x, y, \dot{x}, \dot{y})] F_{1}(x, y, \dot{x}, \dot{y})[d y \delta x-d x \delta y] .
$$

3. Transformation of $F_{1}(x, y, \dot{x}, \dot{y})$. Here a transformation of the variables $(x, y, \dot{x}, \dot{y})$, with $\dot{x}^{2}+\dot{y}^{2}=1$, is defined and the altered form of $F_{1}(x, y, \dot{x}, \dot{y})$ is determined. The existence of the transformation will be assured by invoking a "field hypothesis," (3.4).

Denote by $E$ an arbitrarily directed lineal element at the origin, which makes a positive angle $\theta$ with the positive $X$-axis, and assign to the geodesic tangent to $E$ the orientation of $E$. Let this extremal be defined by the parametric equations

$$
x=X(0, \theta, p), \quad y=Y(0, \theta, p)
$$$$
(0 \leqq \theta<2 \pi),
$$

where the parameter $p$ is signed euclidean arc length, measured from the origin along the geodesic, the sign being positive or negative according as the sense of measurement coincides with the orientation of the extremal or not.

Consider the extremal which is transverse to the geodesic (3.1) at the point (3.1). Orient it by defining its positive sense to be from the point (3.1) to the positive side, the left, of the geodesic (3.1). This extremal will be defined by parametric equations

$$
x=X(s, \theta, p), \quad y=Y(s, \theta, p),
$$

the parameter $s$ being signed euclidean arc length, where

$$
(d X / d s)^{2}+(d Y / d s)^{2}=1,
$$

measured from the point (3.1) along the curve (3.2) and, as above, the sign being positive or negative according as the sense of measurement coincides with the orientation of the extremal or not.

The need for certain field properties, holding in the large for the geodesics (3.2), forces us to postulate the following:

(3.4) FIELD hypothesis. There is a neighborhood, $U$, of the origin which possesses the following properties: (a) $U$ is extremal convex in the large. (b) Any direct lineal element of $U$ is tangent to a unique equally directed extremal (3.2). (c) The jacobians

$$
J_{1}=J_{1}\left(\frac{X, Y, X_{s}}{s, \theta, p}\right), \quad J_{2}=J_{2}\left(\frac{X, Y, Y_{s}}{s, \theta, p}\right)
$$


never vanish simultaneously on $U$.

Hence, a unique triplet $(s, \theta, p)$ is associated with any given directed lineal element $(x, y, \dot{x}, \dot{y})$ of $U$, and

$$
\begin{aligned}
& x=X(s, \theta, p) \quad \dot{x} / \rho=X_{s}(s, \theta, p), \\
& y=Y(s, \theta, p) \quad \dot{y} / \rho=Y_{s}(s, \theta, p) \text {, } \\
& \rho=\left(x^{2}+y^{2}\right)^{1 / 2} .
\end{aligned}
$$

Consequently,

$$
F_{1}=F_{1}\left(X, Y, X_{s}, Y_{s}\right)
$$

is a function of $(s, \theta, p)$ which when partially differentiated with respect to $s$ yields-recalling (1.9)-

$$
\frac{d F_{1}}{d s}=X_{8} F_{1 x}+Y_{8} F_{1 y}+G\left[X_{8} F_{1 \dot{y}}-Y_{8} F_{1 \dot{x}}\right] .
$$

Therefore, equation (1.12) becomes

$$
\frac{d F_{1}}{d s}+\left(X_{s} G_{\dot{y}}-Y_{s} G_{\dot{x}}\right) F_{1}=0,
$$

yielding

$$
F_{1}\left(X, Y, X_{s}, Y_{s}\right)=M(\theta, p) \exp \left[-\int_{0}^{s}\left(X_{s} \dot{G}_{\dot{y}}-Y_{s} G_{\dot{x}}\right) d s\right],
$$

for suitably chosen $M=M(\theta, p)$. Finally, because of (1.4),

$$
F_{1}(x, y, \dot{x}, \dot{y})=\frac{M(\theta, p)}{\rho^{3}} \exp \left[-\int_{0}^{0}\left(X_{s} G_{\dot{y}}-Y_{s} G_{\dot{x}}\right) d s\right]
$$

for arbitrary positive $\rho$.

4. Evaluations for $J_{1}$ and $J_{2}$. By reason of (1.9) the geodesics (3.2) are integral curves for the following differential equations:

$$
X_{s s}=-Y_{s} G\left(X, Y, X_{s}, Y_{s}\right), \quad Y_{s s}=X_{s} G\left(X, Y, X_{s}, Y_{s}\right) .
$$

It follows that

$$
\begin{aligned}
J_{1}\left(\frac{X, Y, X_{s}}{s, \theta, p}\right)= & Y_{s} G\left[X_{p} Y_{\theta}-X_{\theta} Y_{p}\right]+X_{s \theta}\left[X_{p} Y_{s}-X_{s} Y_{p}\right] \\
& +X_{s p}\left[X_{s} Y_{\theta}-X_{\theta} Y_{s}\right] \\
J_{2}\left(\frac{X, Y, Y_{s}}{s, \theta, p}\right)= & X_{s} G\left[Y_{p} X_{\theta}-X_{p} Y_{\theta}\right]+Y_{s \theta}\left[X_{p} Y_{s}-X_{s} Y_{p}\right] \\
& +Y_{s p}\left[X_{s} Y_{\theta}-X_{\theta} Y_{s}\right] .
\end{aligned}
$$


We now deduce from (3.3) that

$$
X_{s} X_{s p}+Y_{s} Y_{s p}=0
$$

and

$$
X_{s} X_{s \theta}+Y_{s} Y_{s \theta}=0,
$$

which imply

$$
X_{s p} Y_{s \theta}-X_{s \theta} Y_{s p}=0 .
$$

These above equations immediately yield

$$
X_{8} J_{1}+Y_{8} J_{2}=0 .
$$

We shall now show that $\left(Y_{s} J_{1}-X_{s} J_{2}\right)$ is the solution of a certain differential equation. A rather tedious direct calculation shows that

$$
\begin{aligned}
\frac{\partial J_{1}}{\partial s}= & \left(X_{s} G_{\dot{y}}-Y_{s} G_{\dot{x}}\right) J_{1}+X_{s} G\left(X_{p} X_{s \theta}+Y_{p} Y_{s \theta}-Y_{\theta} Y_{s p}-X_{\theta} X_{s p}\right) \\
& +X_{s} G^{2}\left(X_{p} Y_{\theta}-X_{\theta} Y_{p}\right)
\end{aligned}
$$

where the simplification only involves using (4.4), (4.5), and (4.6). Another like calculation shows that

$$
\begin{aligned}
\frac{\partial J_{2}}{\partial s}= & \left(X_{s} G_{\dot{y}}-Y_{s} G_{\dot{x}}\right) J_{2}+Y_{s} G\left(Y_{p} Y_{s \theta}+X_{p} X_{s \theta}-Y_{\theta} Y_{s p}-X_{\theta} X_{s p}\right) \\
& -Y_{s} G^{2}\left(Y_{p} X_{\theta}-X_{p} Y_{\theta}\right) .
\end{aligned}
$$

We now infer from (4.8) and (4.9) the relation

$$
Y_{s} \frac{\partial J_{1}}{\partial s}-X_{s} \frac{\partial J_{2}}{\partial s}=\left(X_{s} G_{\dot{y}}-Y_{s} G_{\dot{x}}\right)\left(Y_{s} J_{1}-X_{s} J_{2}\right),
$$

and from (4.1) and (4.7)

$$
\frac{\partial}{\partial s}\left(Y_{s} J_{1}-X_{s} J_{2}\right)=Y_{s} \frac{\partial J_{1}}{\partial s}-X_{s} \frac{\partial J_{2}}{\partial s} .
$$

Thus, because of (4.10) and (4.11),

$$
\frac{\partial}{\partial s}\left(Y_{s} J_{1}-X_{s} J_{2}\right)=\left(X_{s} G_{\dot{y}}-Y_{s} G_{\dot{x}}\right)\left(Y_{s} J_{1}-X_{\diamond} J_{2}\right),
$$

which implies

$$
Y_{\theta} J_{1}-X_{\theta} J_{2}=N(\theta, p) \exp \left[\int_{0}^{s}\left(X_{8} G_{\dot{y}}-Y_{8} G_{\dot{x}}\right) d s\right],
$$


for suitably chosen $N=N(\theta, p)$.

An important relation immediately derivable from (3.9) and (4.13) is

$$
F_{1}(x, y, \dot{x}, \dot{y})=\frac{M(\theta, p) N(\theta, p)}{\rho^{3}\left[Y_{s} J_{1}-X_{s} J_{2}\right]} .
$$

Since, by hypothesis, $F_{1}(x, y, \dot{x}, \dot{y})>0$ and $\left(Y_{s} J_{1}-X_{s} J_{2}\right) \neq 0$, (4.14) implies that the density $M N \neq 0$.

5. A contact transformation. Henceforth, the simple, closed, regular curve $C:\left[(x(t), y(t)), t_{0} \leqq t \leqq t_{1}\right]$ will be traced in the positive sense, that is, $C$ is so traversed, for increasing values of $t$, that its finite component is to the left. Because of the "field hypothesis," associated with $(x, y, \dot{x}, \dot{y})$ is a unique triplet $(s, \theta, p)$, and

$$
\begin{array}{lll}
x=X(s, \theta, p), & \dot{x}=\rho X_{s}(s, \theta, p), & \rho=\left(x^{2}+y^{2}\right)^{1 / 2} . \\
y=Y(s, \theta, p), & \dot{y}=\rho Y_{s}(s, \theta, p), &
\end{array}
$$

An easily established consequence of (5.1) is

$$
\dot{\theta}=\tau\left[X_{s} Y_{p}-X_{p} Y_{s}\right], \quad \dot{p}=\tau\left[X_{\theta} Y_{s}-X_{s} Y_{\theta}\right],
$$

where

$$
\tau=\frac{(\rho-\dot{s})}{\left[X_{\theta} Y_{p}-Y_{\theta} X_{p}\right]} .
$$

Equations (5.1) and (5.2) define a contact transformation of directed lineal elements $(x, y, \dot{x}, \dot{y}) \subset U$ into lineal elements $(\theta, p, \dot{\theta}, \dot{p})$. Furthermore, as seen in $\S 8$, any regular, extremal convex curve $C$ is mapped by these equations into a regular curve $F^{*}:\left[(\theta(t), p(t)), t_{0} \leqq t \leqq t_{1}\right]$ in the cartesian $(\theta, p)$-plane.

6. Transformation of $\delta I$. The altered form of $\delta I,(2.5)$, under the contact transformation will now be computed.

Relations (1.9), (5.1), and (5.2) imply

$$
\begin{aligned}
(\dot{x} \ddot{y}-\dot{y} \ddot{x})= & \rho^{2} \dot{s} G\left(X, Y, X_{s}, Y_{s}\right) \\
& +\rho^{2} \tau\left[X_{s p}\left(Y_{\theta} Y_{s} X_{s}-Y_{s}^{2} X_{\theta}\right)+X_{s \theta}\left(X_{p} Y_{s}^{2}-X_{s} Y_{p} Y_{s}\right)\right] \\
& +\rho^{2} \tau\left[Y_{s p}\left(Y_{s} X_{\theta} X_{s}-Y_{\theta} X_{s}^{2}\right)+Y_{s \theta}\left(X_{s}^{2} Y_{p}-X_{p} Y_{s} X_{s}\right)\right] .
\end{aligned}
$$

Now, computing the value of $X_{8} J_{2}-Y_{8} J_{1}$ and using it in the simplification of (6.1) yields

$$
(\dot{x} \ddot{y}-\dot{y} \ddot{x})=\rho^{2}\left[\dot{s}+\tau\left(X_{\theta} Y_{p}-X_{p} Y_{\theta}\right)\right] G+\tau \rho^{2}\left[Y_{s} J_{1}-X_{8} J_{2}\right] .
$$

Finally, by reason of (1.8) and (5.3), (6.2) reduces to

$$
(\dot{x} \ddot{y}-\dot{y} \ddot{x})=G(x, y, \dot{x}, \dot{y})+\tau \rho^{2}\left[Y_{s} J_{1}-X_{s} J_{2}\right] .
$$

Consequently, by reason of $(2.5),(4.14),(5.1)$, and (6.3), the variation 
$\delta I$ can be expressed as

$$
\delta I=\int_{F^{*}} M(\theta, p) N(\theta, p) \tau\left[\frac{Y_{s} \delta x-X_{s} \delta y}{\left(\dot{\theta}^{2}+\dot{p}^{2}\right)^{1 / 2}}\right]\left((d \theta)^{2}+(d p)^{2}\right)^{1 / 2} .
$$

7. Map of admissible curves $C^{*}$. If the admissible curve $C^{*} \subset U$ encloses the origin, a restriction which is removed in $\$ 9$, then, as we now show, the map of $C^{*}$, under the contact transformation, is a regular arc $F^{*}:\left[(\theta(t), p(t)), t_{0} \leqq t \leqq t_{1}\right]$, in the cartesian $(\theta, p)$-plane, bounded by end points $Q_{0} \equiv\left(\theta\left(t_{0}\right), p\left(t_{0}\right)\right)$, and $Q_{1} \equiv\left(\theta\left(t_{1}\right), p\left(t_{1}\right)\right)=\left(\theta\left(t_{0}\right)+2 \pi, p\left(t_{0}\right)\right)$.

The end point condition for $F^{*}$ is automatically fulfilled, because of the nature of the contact transformation. Furthermore, the regularity of the arc $F^{*}$ follows from (5.2) if it can be asserted that

$$
\left[X_{8} Y_{p}-X_{p} Y_{8}\right]^{2}+\left[X_{\theta} Y_{s}-X_{8} Y_{\theta}\right]^{2} \neq 0
$$

and

$$
\tau \neq 0 \text {. }
$$

The negation of (7.1) gives

$$
X_{s} Y_{\theta}-X_{\theta} Y_{s}=0, \quad X_{s} Y_{p}-X_{p} Y_{s}=0,
$$

which, in turn, implies

$$
X_{\theta} Y_{p}-Y_{\theta} X_{p}=0,
$$

since $X_{s}^{2}+Y_{s}^{2}=1$. Therefore, $J_{1}$ and $J_{2}$, see (4.2) and (4.3), both vanish, which contradicts the "field hypothesis."

We now establish (7.2). Extremal convexity of $C^{*}$ implies that on $C^{*}$

$$
\frac{\dot{x} \ddot{y}-\dot{y} \ddot{x}}{\rho^{3}}-G\left(x, y, \frac{\dot{x}}{\rho}, \frac{\dot{y}}{\rho}\right) \neq 0 .
$$

Consequently, because of (6.3), $\tau \neq 0$ on $C^{*}$.

Furthermore, if $C^{*}$ is extremal convex in the large, then the image $F^{*}$ is a simple arc and has the unique representation $p=p(\theta)$.

8. Double integral with variation $\delta I$. Let

$$
K\left(D^{*}\right) \equiv-\iint_{\Omega} n\left(\theta, p, D^{*}\right) M(\theta, p) N(\theta, p) d p d \theta,
$$

where $\Omega$ is the entire cartesian $(\theta, p)$-space and $n\left(\theta, p, D^{*}\right)$ is the topological order of the point $(\theta, p)$ with respect to the closed oriented curve $D^{*}$. The existence of a $D^{*} \supset F^{*}$ will be established for which the variation of $I\left(C^{*}\right)$ is identical with the induced variation of $K\left(D^{*}\right)$.

$D^{*}$ is defined as follows The contact transformation maps $C^{*}$ into $F^{*}$ with an orientation induced by that of $C^{*}$. The projections of the end points 
of $F^{*}, Q_{0}$ and $Q_{1}$, on the $\theta$-axis will be denoted by $R_{0} \equiv\left(\theta\left(t_{0}\right), 0\right)$ and $R_{1}$ $\equiv\left(\theta\left(t_{1}\right), 0\right)$. Then, $D^{*}$ is the oriented curvilinear polygon consisting of the directed line-segments $Q_{1} R_{1}, R_{1} R_{0}, R_{0} Q_{0}$ and the directed arc $F^{*}$.

The verification of $\delta I\left(C^{*}\right)=\delta K\left(D^{*}\right)$ is now considered. Embed $F^{*} \equiv F_{0}^{*}$ in a 1-parameter family of curves

$$
F_{k}^{*}: \begin{array}{lll}
\theta=\theta(t, k) ; & t_{0} \leqq t \leqq t_{1} ; & \theta\left(t_{0}, k\right)=\theta\left(t_{0}\right), \\
p=p(t, k) ; & 0 \leqq k \leqq k_{0} ; & \theta\left(t_{1}, k\right)=\theta\left(t_{1}\right),
\end{array}
$$

for sufficiently small $k_{0}$. Introduce the notation $K(k) \equiv K\left(D_{k}^{*}\right)$, where $D_{k}^{*}$ and $F_{k}^{*}$ are related in the same manner as $D^{*}$ and $F^{*}$, and observe that since $D_{0}^{*}$ has at most a finite number of singularities,

$$
\lim _{\epsilon \rightarrow 0}\left[n\left(\theta, p, D_{\epsilon}^{*}\right)-n\left(\theta, p, D_{0}^{*}\right)\right]=\operatorname{sign}\left[\delta p \theta_{t}-\delta \theta p_{t}\right],
$$

where $(\delta \theta, \delta p) \equiv\left(\theta_{k}(t, 0), p_{k}(t, 0)\right)$. Consequently,

$$
\begin{aligned}
\delta K\left(D_{0}^{*}\right) & \equiv \lim _{\epsilon \rightarrow 0} \frac{K(\epsilon)-K(0)}{\epsilon} \\
& =-\int_{F_{0}^{*}} M(\theta, p) N(\theta, p)\left[\frac{\delta p \theta_{t}-\delta \theta p_{t}}{\left(\dot{\theta}^{2}+\dot{p}^{2}\right)^{1 / 2}}\right]\left((d \theta)^{2}+(d p)^{2}\right)^{1 / 2} .
\end{aligned}
$$

Therefore, by reason of (6.4) and (8.4), $\delta I\left(C^{*}\right)=\delta K\left(D^{*}\right)$ if

$$
\tau\left[\frac{Y_{\delta} \delta x-X_{s} \delta y}{\left(\dot{\theta}^{2}+\dot{p}^{2}\right)^{1 / 2}}\right]=\left[\frac{\delta \theta p_{t}-\delta p \theta_{t}}{\left(\dot{\theta}^{2}+\dot{p}^{2}\right)^{1 / 2}}\right],
$$

where $(\delta \theta, \delta p)$ is the variation induced in $(\theta(t), p(t))$ by the variation $(\delta x, \delta y)$ of $(x(t), y(t))$. We now consider the proof of (8.5). Since

$$
\begin{aligned}
& \delta x=\delta s X_{s}+\delta \theta X_{\theta}+\delta p X_{p} \quad \text { and } \\
& \delta y=\delta s Y_{s}+\delta \theta Y_{\theta}+\delta p Y_{p},
\end{aligned}
$$

it follows that

$$
Y_{s} \delta x-X_{s} \delta y=\left(Y_{s} X_{p}-X_{s} Y_{p}\right) \delta p+\left(Y_{s} X_{\theta}-X_{s} Y_{\theta}\right) \delta \theta .
$$

Thus, because of (5.2),

$$
Y_{8} \delta x-X_{s} \delta y=\left[\frac{\delta \theta p_{t}-\delta p \theta_{t}}{\tau}\right],
$$

which completes the verification of (8.5).

9. The identity $I\left(C^{*}\right)=K\left(D^{*}\right)$. We now impose the following condition:

(9.1) EMBedment hypothesis. The admissible curves $C^{*}$ can be embedded in a 1-parameter family of closed curves $C_{k}^{*}:\left[(x(t, k), y(t, k)) ; t_{0} \leqq t \leqq t_{1}\right.$, 
$0<k \leqq 1]$ which possess the following properties: (a) $C_{1}^{*} \equiv C^{*}$ (b) The $C_{k}^{*}$ are admissible curves which are sufficiently differentiable in the variables $(t, k)$. (c) $C_{k}^{*}$ tends uniformly to the origin as $k$ tends to zero. (d) The map $F_{k}^{*}$ of $C_{k}^{*}$, under the contact transformation, is such that $\theta\left(t_{0}, k\right)$ and $\theta\left(t_{1}, k\right)$ are independent of $k$.

The identity will now be proved. Since the curves (3.2) were so defined that $X(0, \theta, 0)=Y(0, \theta, 0)=0$, the "field and embedment hypothesis" imply that $D_{k}^{*}$ tends uniformly to a finite segment on the $\theta$-axis, as $k$ tends to zero. Therefore, since $\delta I=\delta K$,

$$
I\left(C^{*}\right)=\lim _{k \rightarrow 0} \int_{k}^{1} \delta I d k=\lim _{k \rightarrow 0} \int_{k}^{1} \delta K d k=K\left(D^{*}\right),
$$

which is the same as

$$
\oint_{C^{*}} F(x, y, d x, d y)=-\iint_{\Omega} n\left(\theta, p, D^{*}\right) M(\theta, p) N(\theta, p) d p d \theta ;
$$

and, if $C^{*}$ is extremal convex in the large, (9.3) reduces to

$$
\oint_{C^{*}} F(x, y, d x, d y)=-\iint_{D} M(\theta, p) N(\theta, p) d p d \theta,
$$

since $F^{*}$ now admits the unique, continuously differentiable, nonparametric representation $p=p(\theta)$.

If $C^{*}$ does not enclose the origin, $C^{*}$ will lie in a curvilinear sector defined by two tangent geodesics (3.1) having minimum and maximum values of $\theta$. If an "embedment hypothesis" can be invoked which guarantees the contraction of $C^{*}$ in the sector to the origin by means of admissible curves whose end points lie on the bounding tangents, then the validity of (9.3) and (9.4) is assured.

10. Results for special metrics. If the geodesics of the metric are straight lines, then

$$
\begin{aligned}
& x=X(s, \theta, p)=p \cos \theta-s \sin \theta \\
& y=Y(s, \theta, p)=p \sin \theta+s \cos \theta
\end{aligned}
$$

and

$$
\oint_{C^{*}} F(x, y, d x, d y)=\iint_{D} \rho^{3} F_{1}(x, y, \dot{x}, \dot{y}) d p d \theta
$$

Let

$$
2 u=(1 / p+p), \quad 2 v=(1 / p-p),
$$

and $C^{*} C U: x^{2}+y^{2}<1$. Then, for the hyperbolic metric, 
(10.3)

$$
\begin{aligned}
& x=X(s, \theta, p)=u \cos \theta-v \cos (s / v-\theta), \\
& v=Y(s, \theta, p)=u \sin \theta+v \sin (s / v-\theta),
\end{aligned}
$$

and

(10.4) $\quad \oint_{C^{*}} \frac{\left((d x)^{2}+(d y)^{2}\right)^{1 / 2}}{1-x^{2}-y^{2}}=\iint_{D} \frac{1+p^{2}}{\left(1-p^{2}\right)^{2}} d p d \theta$;

while in the case of the spherical metric,

$$
\begin{aligned}
& x=X(s, \theta, p)=-v \cos \theta+u \cos (s / u+\theta), \\
& y=Y(s, \theta, p)=-v \sin \theta+u \sin (s / u+\theta),
\end{aligned}
$$

and

$$
\oint_{C^{*}} \frac{\left((d x)^{2}+(d y)^{2}\right)^{1 / 2}}{1+x^{2}+y^{2}}=\iint_{D} \frac{1-p^{2}}{\left(1+p^{2}\right)^{2}} d p d \theta .
$$

UNIVERSITY OF WisCONSIN, Madison, Wis. 